\title{
On identities with multinomial coefficients for Fibonacci-Narayana sequence
}

\author{
Taras Goy \\ Vasyl Stefanyk Precarpathian National University, 76018 Ivano-Frankivsk, Ukraine \\ tarasgoy@yahoo.com
}

Submitted August 4, 2017 - Accepted September 25, 2018

\begin{abstract}
In this paper we study some families of Toeplitz-Hessenberg determinants the entries of which are Fibonacci-Narayana (or Narayana's cows) numbers. This leads to discover some identities for these numbers. In particular, we establish connection between Fibonacci-Narayana numbers with Fibonacci and tribonacci numbers. We also present new formulas for Fibonacci-Narayana numbers via recurrent determinants of four-diagonal matrix.
\end{abstract}

Keywords: Fibonacci-Narayana sequence, Narayana's cow sequence, ToeplitzHessenberg matrix, Fibonacci sequence, tribonacci sequence.

$M S C$ : AMS classification 11B39, $15 \mathrm{~B} 05$.

\section{Introduction}

The Fibonacci sequence $\left\{F_{n}\right\}_{n \geq 0}$ is defined by the initial values $F_{0}=0, F_{1}=1$ and the recurrence relation $F_{n}=F_{n-1}+F_{n-2}$, where $n \geq 2$.

Among the several generalizations of Fibonacci numbers, some of the best known are the tribonacci sequence $\left\{t_{n}\right\}_{n \geq 0}$ and the Fibonacci-Narayana sequence (or Narayana's cows sequence) $\left\{b_{n}\right\}_{n \geq 0}$, which are defined by the following thirdorder recurrence relations:

$$
\begin{aligned}
t_{n}=t_{n-1}+t_{n-2}+t_{n-3}, & t_{0}=t_{1}=0, t_{2}=1, \\
b_{n}=b_{n-1}+b_{n-3}, & b_{0}=0, b_{1}=b_{2}=1,
\end{aligned}
$$

for $n \geq 3$. 
There are large number of sequences indexed in OEIS [10], being in this case

$$
\begin{array}{rll}
\left\{F_{n}\right\}_{n \geq 0} & =\{0,1,1,2,3,5,8,13,21,34,55,89,144,233,377, \ldots\}: & A 000045 \\
\left\{t_{n}\right\}_{n \geq 0}=\{0,0,1,1,2,4,7,13,24,44,81,149,274,504,927, \ldots\}: & A 000073 \\
\left\{b_{n}\right\}_{n \geq 0}=\{0,1,1,1,2,3,4,6,9,13,19,28,41,60,88,129, \ldots\}: & A 000930
\end{array}
$$

The Fibonacci-Narayana sequence was introduced by the Indian mathematician Narayana in the 14th century, while studying the following problem: $A$ cow produces one calf every year. Beginning in its fourth year, each calf produces one calf at the beginning of each year. How many cows are there altogether after, for example, 20 years? This problem can be solved in the same way that Fibonacci solved its problem about rabbits [6].

There has been considerable recent interest in the Fibonacci-Narayana sequence and its generalizations (see $[1,2,3,4,5,9,11]$ for more details). For instance, Didkivska and St'opochkina [4] proved some basic properties of Fibonacci-Narayana numbers. Biglici [3] defined a generalized order- $k$ Fibonacci-Narayana sequence and by using this generalization and some matrix properties, established some identities related to Fibonacci-Narayana numbers. Flaut and Shpakivskyi [5] studied some properties of generalized and Fibonacci quaternions and Fibonacci-Narayana quaternions. Ramírez and Sirvent [9] defined the $k$-Narayana sequence of integer numbers and studied recurrence relations and some combinatorial properties of these numbers, and of the sum of their first $n$ terms. These authors also established some relations between the $k$-Narayana sequence and determinants of one type of Hessenberg matrix.

The purpose of this paper is to study Fibonacci-Narayana numbers. We investigate some families of Toeplitz-Hessenberg determinants the entries of which are Fibonacci-Narayana numbers. This leads to discover some identities for these numbers. In particular, we establish connection between Fibonacci-Narayana numbers with Fibonacci and tribonacci numbers. We also present new formulas for Fibonacci-Narayana numbers via recurrent determinants of four-diagonal matrix.

\section{Toeplitz-Hessenberg matrices and determinants}

A Toeplitz-Hessenberg matrix is an $n \times n$ matrix of the form

$$
M_{n}\left(a_{0} ; a_{1}, a_{2}, \ldots, a_{n}\right)=\left[\begin{array}{cccccc}
a_{1} & a_{0} & 0 & \ldots & 0 & 0 \\
a_{2} & a_{1} & a_{0} & \ldots & 0 & 0 \\
a_{3} & a_{2} & a_{1} & \ldots & 0 & 0 \\
\ldots & \ldots & \ldots & \ddots & \ldots & \ldots \\
a_{n-1} & a_{n-2} & a_{n-3} & \ldots & a_{1} & a_{0} \\
a_{n} & a_{n-1} & a_{n-2} & \ldots & a_{2} & a_{1}
\end{array}\right]
$$

where $a_{0} \neq 0$ and $a_{k} \neq 0$ for at least one $k>0$. So $a_{i j}=0$ for $j>i+1$.

Several mathematical objects may be represented as determinant of such matrices (see, for example, [7] and the references given there). 
Expanding the determinant $\operatorname{det}\left(M_{n}\right)$ according to the first row repeatedly, we obtain the recurrence

$$
\operatorname{det}\left(M_{n}\right)=\sum_{k=1}^{n}\left(-a_{0}\right)^{k-1} a_{k} \operatorname{det}\left(M_{n-k}\right)
$$

where, by definition, $\operatorname{det}\left(M_{0}\right) \equiv 1$.

The following result is known as Trudi's formula [8]. This gives the multinomial extension for $\operatorname{det}\left(M_{n}\right)$.

Lemma 2.1. Let $n$ be a positive integer. Then

$$
\operatorname{det}\left(M_{n}\right)=\sum_{\left(s_{1}, \ldots, s_{n}\right)}\left(-a_{0}\right)^{n-\left(s_{1}+\cdots+s_{n}\right)}\left(\begin{array}{c}
s_{1}+\cdots+s_{n} \\
s_{1}, \ldots, s_{n}
\end{array}\right) a_{1}^{s_{1}} a_{2}^{s_{2}} \cdots a_{n}^{s_{n}}
$$

where the summation is over integers $s_{i} \geq 0$ satisfying $s_{1}+2 s_{2}+\cdots+n s_{n}=n$, and

$$
\left(\begin{array}{c}
s_{1}+\cdots+s_{n} \\
s_{1}, \ldots, s_{n}
\end{array}\right)=\frac{\left(s_{1}+\cdots+s_{n}\right) !}{s_{1} ! \cdots s_{n} !}
$$

is the multinomial coefficient.

Example 2.2. It follows from (2.3) that

$$
\begin{aligned}
\operatorname{det}\left(M_{3}\right)= & \left(-a_{0}\right)^{0}\left(\begin{array}{c}
3 \\
3,0,0
\end{array}\right) a_{1}^{3}+\left(-a_{0}\right)^{1}\left(\begin{array}{c}
2 \\
1,1,0
\end{array}\right) a_{1} a_{2}+\left(-a_{0}\right)^{2}\left(\begin{array}{c}
1 \\
0,0,1
\end{array}\right) a_{3} \\
= & a_{1}^{3}-2 a_{0} a_{1} a_{2}+a_{0}^{2} a_{3} \\
\operatorname{det}\left(M_{4}\right)= & \left(-a_{0}\right)^{0}\left(\begin{array}{c}
4 \\
4,0,0,0
\end{array}\right) a_{1}^{4}+\left(-a_{0}\right)^{1}\left(\begin{array}{c}
3 \\
2,1,0,0
\end{array}\right) a_{1}^{2} a_{2}+\left(-a_{0}\right)^{2}\left(\begin{array}{c}
2 \\
1,0,1,0
\end{array}\right) a_{1} a_{3} \\
& +\left(-a_{0}\right)^{2}\left(\begin{array}{c}
2 \\
0,2,0,0
\end{array}\right) a_{2}^{2}+\left(-a_{0}\right)^{3}\left(\begin{array}{c}
1 \\
0,0,0,1
\end{array}\right) a_{4} \\
= & a_{1}^{4}-3 a_{0} a_{1}^{2} a_{2}+2 a_{0}^{2} a_{1} a_{3}+a_{0}^{2} a_{2}^{2}-a_{0}^{3} a_{4} .
\end{aligned}
$$

Throughout this paper, we denote

$$
\operatorname{det}\left( \pm 1 ; a_{1}, a_{2}, \ldots, a_{n}\right)=\operatorname{det}\left(M_{n}\left( \pm 1 ; a_{1}, a_{2}, \ldots, a_{n}\right)\right) .
$$

\section{Connection formulas between the Fibonacci-Narayana numbers and Fibonacci numbers}

The next theorem gives relationship between Fibonacci-Narayana numbers and Fibonacci numbers via the Toeplitz-Hessenberg determinants. 
Theorem 3.1. For all $n \geq 1$,

$$
\begin{aligned}
& \operatorname{det}\left(1 ; b_{1}, b_{3}, \ldots, b_{2 n-1}\right)=1-(-1)^{n} F_{n-1}, \\
& \operatorname{det}\left(1 ; b_{0}, b_{2}, \ldots, b_{2 n-2}\right)=(-1)^{n-1} F_{n} .
\end{aligned}
$$

Proof. We will prove formula (3.1) using induction on $n$. The other proof follow similarly, so we omit it for interest of brevity. For simplicity of notation, we write $D_{n}$ instead of $\operatorname{det}\left(1 ; b_{1}, b_{3}, \ldots, b_{2 n-1}\right)$.

Clearly, formula (3.1) works, when $n=1$ and $n=2$. Suppose it is true for all positive integers $k \leq n-1$, where $n \geq 3$.

Using recurrence (2.2), we have

$$
\begin{aligned}
D_{n}= & \sum_{i=1}^{n}(-1)^{i-1} b_{2 i-1} D_{n-i} \\
= & b_{1} D_{n-1}-b_{3} D_{n-2}+b_{5} D_{n-3}+\sum_{i=4}^{n}(-1)^{i-1}\left(b_{2 i-2}+b_{2 i-4}\right) D_{n-i} \\
= & D_{n-1}-D_{n-2}+3 D_{n-3}+\sum_{i=4}^{n}(-1)^{i-1}\left(b_{2 i-3}+2 b_{2 i-5}+b_{2 i-7}\right) D_{n-i} \\
= & D_{n-1}-D_{n-2}+3 D_{n-3}+\sum_{i=3}^{n-1}(-1)^{i} b_{2 i-1} D_{n-i-1} \\
& +2 \sum_{i=2}^{n-2}(-1)^{i+1} b_{2 i-1} D_{n-i-2}+\sum_{i=1}^{n-3}(-1)^{i+2} b_{2 i-1} D_{n-i-3} \\
= & D_{n-1}-D_{n-2}+3 D_{n-3}+\left(\sum_{i=1}^{n-1}(-1)^{i} b_{2 i-1} D_{n-i-1}+b_{1} D_{n-2}-b_{3} D_{n-3}\right) \\
& +\left(2 \sum_{i=1}^{n-2}(-1)^{i} b_{2 i-1} D_{n-i-2}-2 b_{1} D_{n-3}\right)-D_{n-3} \\
= & D_{n-1}-D_{n-2}+3 D_{n-3}-D_{n-1}+D_{n-2}-D_{n-3}+2 D_{n-2}-2 D_{n-3}-D_{n-3} \\
= & 2 D_{n-2}-D_{n-3} .
\end{aligned}
$$

Using the induction hypothesis and the definition of the Fibonacci sequence, we obtain

$$
\begin{aligned}
D_{n} & =2\left(1-(-1)^{n-2} F_{n-3}\right)-\left(1-(-1)^{n-3} F_{n-4}\right) \\
& =1-(-1)^{n}\left(2 F_{n-3}+F_{n-4}\right) \\
& =1-(-1)^{n} F_{n-1} .
\end{aligned}
$$

Consequently, the formula (3.1) is true for $n$. Therefore, by induction, the formula works for all positive integers $n$. 


\section{Connection formula between the Fibonacci-Narayana numbers and tribonacci numbers}

The next theorem gives relationship between Fibonacci-Narayana numbers and tribonacci numbers via the Toeplitz-Hessenberg determinants.

Theorem 4.1. For all $n \geq 1$,

$$
\operatorname{det}\left(-1 ; b_{0}, b_{1}, \ldots, b_{n-1}\right)=t_{n} .
$$

Proof. We will prove formula (4.1) using induction on $n$. It is easily seen that $\operatorname{det}\left(-1 ; b_{0}, b_{1}, \ldots, b_{n-1}\right)=(-1)^{n} D_{n}$, where

$$
D_{n}=\operatorname{det}\left(1 ;-b_{0},-b_{1}, \ldots,-b_{n-1}\right) .
$$

Clearly, formula (4.1) works, when $n=1$ and $n=2$. Suppose it is true for all $k \leq n-1$, where $n \geq 2$. Using recurrence (2.2), we have

$$
\begin{aligned}
D_{n} & =\sum_{i=1}^{n}(-1)^{i} b_{i-1} D_{n-i} \\
& =-b_{0} D_{n-1}+b_{1} D_{n-2}-b_{2} D_{n-3}+\sum_{i=4}^{n}(-1)^{i}\left(b_{i-2}+b_{i-4}\right) D_{n-i} \\
& =D_{n-2}-D_{n-3}+\sum_{i=3}^{n-1}(-1)^{i+1} b_{i-1} D_{n-i-1}+\sum_{i=1}^{n-3}(-1)^{i+3} b_{i-1} D_{n-i-3} \\
& =D_{n-2}-D_{n-3}+\left(\sum_{i=1}^{n-1}(-1)^{i+1} b_{i-1} D_{n-i-1}-b_{0} D_{n-2}+b_{1} D_{n-3}\right)-D_{n-3} \\
& =D_{n-2}-D_{n-3}-D_{n-1}+D_{n-3}-D_{n-3} \\
& =-D_{n-1}+D_{n-2}-D_{n-3} .
\end{aligned}
$$

Thus,

$$
\begin{aligned}
\operatorname{det}\left(1 ;-b_{0},-b_{1}, \ldots,-b_{n-1}\right) & =(-1)^{n}\left(-\frac{t_{n-1}}{(-1)^{n-1}}+\frac{t_{n-2}}{(-1)^{n-2}}-\frac{t_{n-3}}{(-1)^{n-3}}\right) \\
& =t_{n-1}+t_{n-2}+t_{n-3}=t_{n} .
\end{aligned}
$$

Consequently, the formula (4.1) is true for $n$. Therefore, by induction, the formula works for all positive integers $n$.

\section{Some Toeplitz-Hessenberg determinants with Fibonacci-Narayana entries}

In this section, we evaluate $\operatorname{det}\left( \pm 1 ; a_{1}, a_{2}, \ldots, a_{n}\right)$ with special Fibonacci-Narayana entries $a_{i}$. 
Theorem 5.1. Let $n \geq 1$, except when noted otherwise. Then

$$
\begin{aligned}
& \operatorname{det}\left(1 ; b_{0}, b_{1}, \ldots, b_{n-1}\right)=\frac{(-1)^{n-1}+(-1)^{\left\lfloor\frac{n+1}{2}\right\rfloor}}{2}, \\
& \operatorname{det}\left(1 ; b_{1}, b_{2}, \ldots, b_{n}\right)=\frac{(-1)^{n-1}+(-1)^{\lfloor n / 3\rfloor}}{2}, \\
& \operatorname{det}\left(-1 ; b_{1}, b_{2}, \ldots, b_{n}\right)=2^{n-1} \sum_{i=0}^{\left\lfloor\frac{n-1}{3}\right\rfloor} \frac{1}{8^{i}}\left(\begin{array}{c}
n-1-2 i \\
i
\end{array}\right) \text {, } \\
& \operatorname{det}\left(1 ; b_{2}, b_{3}, \ldots, b_{n+1}\right)=0, \quad n \geq 4 \text {, } \\
& \operatorname{det}\left(1 ; b_{2}, b_{4}, \ldots, b_{2 n}\right)=(-1)^{n-1} \sum_{i=0}^{n}\left(\begin{array}{c}
i+1 \\
n-1-2 i
\end{array}\right) \text {, } \\
& \operatorname{det}\left(-1 ; b_{2}, b_{4}, \ldots, b_{2 n}\right)=\sum_{i=0}^{2 n-1}\left(\begin{array}{c}
i \\
4 n-1-2 i
\end{array}\right), \\
& \operatorname{det}\left(1 ; b_{3}, b_{4}, \ldots, b_{n+2}\right)=(-1)^{\frac{n}{2}}\left(1+(-1)^{n}\right) / 2, \quad n \geq 2 \text {, } \\
& \operatorname{det}\left(1 ; b_{3}, b_{5}, \ldots, b_{2 n+1}\right)=0, \quad n \geq 4 \text {, } \\
& \operatorname{det}\left(1 ; b_{4}, b_{5}, \ldots, b_{n+3}\right)=\frac{(-1)^{\left\lfloor\frac{n-1}{3}\right\rfloor}+(-1)^{\left\lfloor\frac{n}{3}\right\rfloor}}{2}, \\
& \operatorname{det}\left(1 ; b_{4}, b_{6}, \ldots, b_{2 n+2}\right)=1, \quad n \geq 3, \\
& \operatorname{det}\left(1 ; b_{5}, b_{6}, \ldots, b_{n+4}\right)=\sum_{i=0}^{n+2}\left(\begin{array}{c}
n+2-i \\
1+2 i
\end{array}\right), \\
& \operatorname{det}\left(1 ; b_{5}, b_{7}, \ldots, b_{2 n+3}\right)=n+1, \quad n \geq 2, \\
& \operatorname{det}\left(1 ; b_{6}, b_{8}, \ldots, b_{2 n+4}\right)=\left(n^{2}+3 n+4\right) / 2,
\end{aligned}
$$

where $\left(\begin{array}{c}m \\ k\end{array}\right)=\frac{m !}{k !(m-k) !}$ is the binomial coefficient and $\lfloor\cdot\rfloor$ is the floor function.

Proof. We will prove only (5.1), the other ones can be proved in the same way. Obviously, $\operatorname{det}\left(-1 ; b_{1}, b_{2}, \ldots, b_{n}\right)=(-1)^{n} D_{n}$, where

$$
D_{n}=\operatorname{det}\left(1 ;-b_{1},-b_{2}, \ldots,-b_{n}\right),
$$

When $n=1$ and $n=2$, the formula holds. Assuming (5.1) to hold for all $k \leq n-1$, we prove it for $n \geq 3$. Using (2.2), we have

$$
\begin{aligned}
D_{n} & =\sum_{i=1}^{n}(-1)^{i} b_{i} D_{n-i} \\
& =-b_{1} D_{n-1}+b_{2} D_{n-2}-b_{3} D_{n-3}+\sum_{i=4}^{n}(-1)^{i}\left(b_{i-1}+b_{i-4}\right) D_{n-i} \\
& =-D_{n-1}+D_{n-2}-D_{n-3}+\sum_{i=3}^{n-1}(-1)^{i+1} b_{i} D_{n-1-i}+\sum_{i=1}^{n-3}(-1)^{i+3} b_{i} D_{n-3-i}
\end{aligned}
$$




$$
\begin{aligned}
& =-D_{n-1}+D_{n-2}-D_{n-3}+\sum_{i=1}^{n-1}(-1)^{i+1} b_{i} D_{n-1-i}-D_{n-2}+D_{n-3}-D_{n-3} \\
& =-D_{n-1}+D_{n-2}-D_{n-3}-D_{n-1}-D_{n-2}+D_{n-3}-D_{n-3} \\
& =-2 D_{n-1}-D_{n-3} \\
& =-(-2)^{n-1} \sum_{k=0}^{\left\lfloor\frac{n-2}{3}\right\rfloor} \frac{1}{8^{k}}\left(\begin{array}{c}
n-2-2 k \\
k
\end{array}\right)-(-2)^{n-4} \sum_{k=0}^{\left\lfloor\frac{n-1}{3}\right\rfloor-1} \frac{1}{8^{k}}\left(\begin{array}{c}
n-4-2 k \\
k
\end{array}\right) .
\end{aligned}
$$

Thus,

$$
D_{n}=-(-2)^{n-1} \sum_{k=0}^{\left\lfloor\frac{n-2}{3}\right\rfloor} \frac{1}{8^{k}}\left(\begin{array}{c}
n-2-2 k \\
k
\end{array}\right)-(-2)^{n-1} \sum_{k=1}^{\left\lfloor\frac{n-1}{3}\right\rfloor} \frac{1}{8^{k}}\left(\begin{array}{c}
n-2-2 k \\
k-1
\end{array}\right) .
$$

Let $n \neq 3 m-2$. Then $\left\lfloor\frac{n-2}{3}\right\rfloor=\left\lfloor\frac{n-1}{3}\right\rfloor$. From (5.2), using well-known formula

$$
\left(\begin{array}{c}
n-1 \\
k
\end{array}\right)+\left(\begin{array}{l}
n-1 \\
k-1
\end{array}\right)=\left(\begin{array}{l}
n \\
k
\end{array}\right)
$$

we have

$$
\begin{aligned}
D_{n} & =-(-2)^{n-1} \sum_{k=0}^{\left\lfloor\frac{n-1}{3}\right\rfloor} \frac{1}{8^{k}}\left(\begin{array}{c}
n-2-2 k \\
k
\end{array}\right)-(-2)^{n-1} \sum_{k=1}^{\left\lfloor\frac{n-1}{3}\right\rfloor} \frac{1}{8^{k}}\left(\begin{array}{c}
n-2-2 k \\
k-1
\end{array}\right) . \\
& =-(-2)^{n-1} \sum_{k=0}^{\left\lfloor\frac{n-1}{3}\right\rfloor} \frac{1}{8^{k}}\left(\begin{array}{c}
n-1-2 k \\
k
\end{array}\right) .
\end{aligned}
$$

Let $n=3 m-2$. Then $\left\lfloor\frac{n-1}{3}\right\rfloor-\left\lfloor\frac{n-2}{3}\right\rfloor=1$. Now, using (5.3), we have

$$
\begin{aligned}
D_{n} & =-2^{n-1} \sum_{k=0}^{\left\lfloor\frac{n-1}{3}\right\rfloor-1} \frac{1}{8^{k}}\left(\begin{array}{c}
n-2-2 k \\
k
\end{array}\right)-2^{n-1} \sum_{k=0}^{\left\lfloor\frac{n-1}{3}\right\rfloor} \frac{1}{8^{k}}\left(\begin{array}{c}
n-2-2 k \\
k-1
\end{array}\right) \\
& =-(-2)^{n-1} \sum_{k=0}^{\left\lfloor\frac{n-1}{3}\right\rfloor} \frac{1}{8^{k}}\left(\begin{array}{c}
n-1-2 k \\
k
\end{array}\right) .
\end{aligned}
$$

Therefore,

$$
\begin{aligned}
\operatorname{det}\left(-1 ; b_{1}, b_{2}, \ldots, b_{n}\right) & =(-1)^{n+1}(-2)^{n-1} \sum_{k=0}^{\left\lfloor\frac{n-1}{3}\right\rfloor} \frac{1}{8^{k}}\left(\begin{array}{c}
n-1-2 k \\
k
\end{array}\right) \\
& =2^{n-1} \sum_{k=0}^{\left\lfloor\frac{n-1}{3}\right\rfloor} \frac{1}{8^{k}}\left(\begin{array}{c}
n-1-2 k \\
k
\end{array}\right) .
\end{aligned}
$$

Since the formula (5.1) holds for $n$, it follows by induction that it is true for all positive integers $n$. 
The Trudi formula (2.3), taken together with Theorems 3.1 and 4.1 yields the following corollary. Similarly, one can obtain the multinomial extensions for formulas from Theorem 5.1.

Corollary 5.2. The following formulas hold:

$$
\begin{gathered}
\sum_{2 s_{1}+3 s_{2}+\cdots+n s_{n-1}=n}(-1)^{\sigma_{n-1}} p_{n}(s) b_{2}^{s_{1}} b_{4}^{s_{2}} \cdots b_{2 n-2}^{s_{n-1}}=-F_{n}, \quad n \geq 2, \\
\sum_{s_{1}+2 s_{2}+\cdots+n s_{n}=n}(-1)^{\sigma_{n}} p_{n}(s) b_{1}^{s_{1}} b_{3}^{s_{2}} \cdots b_{2 n-1}^{s_{n}}=(-1)^{n}-F_{n-1}, \quad n \geq 1, \\
\sum_{2 s_{1}+3 s_{2}+\cdots+n s_{n-1}=n} p_{n}(s) b_{1}^{s_{1}} b_{2}^{s_{2}} \cdots b_{n-1}^{s_{n-1}}=t_{n}, \quad n \geq 2,
\end{gathered}
$$

where $p_{n}(s)=\left(\begin{array}{c}s_{1}+\cdots+s_{n} \\ s_{1}, \ldots, s_{n}\end{array}\right)$ is the multinomial coefficient, $\sigma_{n}=s_{1}+\cdots+s_{n}, s_{i} \geq 0$, $F_{n}$ and $t_{n}$ are the $n$-th Fibonacci and tribonacci numbers, respectively.

Example 5.3. It follows from (5.4), (5.5), and (5.6), respectively, that

$$
\begin{aligned}
b_{2}^{3}-2 b_{2} b_{6}-b_{4}^{2}+b_{10} & =F_{6}, \\
b_{1}^{5}-4 b_{1}^{3} b_{3}+3 b_{1}^{2} b_{5}+3 b_{1} b_{3}^{2}-2 b_{1} b_{7}-2 b_{3} b_{5}+b_{9}-1 & =F_{4}, \\
3 b_{1}^{2}+2 b_{1} b_{4}+2 b_{2} b_{3}+b_{6} & =t_{7} .
\end{aligned}
$$

\section{Fibonacci-Narayana determinants}

In this section, we prove two formulas expressing Fibonacci-Narayana numbers $b_{i}$ with even (odd) subscripts via recurrent determinants of four-diagonal matrix of order $n$.

Let $P_{n}$ and $Q_{n}$ denote the $n \times n$ four-diagonal matrices

$$
P_{n}=\left[\begin{array}{ccccccc}
1 & b_{2} & & & & & \\
-2 & 0 & b_{4} & & & 0 & \\
b_{5} & -b_{3} & 0 & b_{6} & & & \\
& b_{7} & -b_{5} & 0 & b_{8} & & \\
& & \ddots & \ddots & \ddots & \ddots & \\
& 0 & & b_{2 n-3} & -b_{2 n-5} & 0 & b_{2 n-2} \\
& & & & b_{2 n-1} & -b_{2 n-3} & 0
\end{array}\right]
$$

and

$$
Q_{n}=\left[\begin{array}{ccccccc}
1 & b_{1} & & & & & \\
-1 & 0 & b_{3} & & & 0 & \\
b_{4} & -b_{2} & 0 & b_{5} & & & \\
& b_{6} & -b_{4} & 0 & b_{7} & & \\
& & \ddots & \ddots & \ddots & \ddots & \\
& 0 & & b_{2 n-4} & -b_{2 n-6} & 0 & b_{2 n-3} \\
& & & & b_{2 n-2} & -b_{2 n-4} & 0
\end{array}\right]
$$


Theorem 6.1. For all $n \geq 1$,

$$
\begin{aligned}
b_{2 n} & =\frac{1}{\prod_{i=1}^{n-1} b_{2 i}} \operatorname{det}\left(P_{n}\right), \\
b_{2 n-1} & =\frac{1}{\prod_{i=1}^{n-1} b_{2 i-1}} \operatorname{det}\left(Q_{n}\right) .
\end{aligned}
$$

Proof. We prove only formula (6.2), the formula (6.1) one can be proved similarly. We use induction on $n$. Since $\operatorname{det}\left(P_{1}\right)=1=b_{2}$ and $\operatorname{det}\left(P_{2}\right)=2=b_{4}$, the result is true when $n=1$ and $n=2$. Assume it true for every positive integer $k<n$. Expanding $\operatorname{det}\left(P_{n}\right)$ by the last row, we have

$$
\begin{aligned}
b_{2 n} & =\frac{1}{\prod_{i=1}^{n-1} b_{2 i}}\left(b_{2 n-3} b_{2 n-2} \operatorname{det}\left(P_{n-2}\right)+b_{2 n-1} b_{2 n-2} b_{2 n-4} \operatorname{det}\left(P_{n-3}\right)\right) \\
= & \frac{1}{\prod_{i=1}^{n-1} b_{2 i}}\left(b_{2 n-3} b_{2 n-2} \prod_{i=1}^{n-2} b_{2 i}+b_{2 n-1} b_{2 n-2} b_{2 n-4} \prod_{i=1}^{n-3} b_{2 i}\right) \\
= & b_{2 n-3}+b_{2 n-1} .
\end{aligned}
$$

Therefore, the result is true for every $n \geq 1$.

Acknowledgements. I would like to thank the referee for a very careful reading of the manuscript and several useful suggestions.

\section{References}

[1] Allouche, J.-P., Johnson, T., Narayana's cows and delayed morphisms, In: Articles of 3rd Computer Music Conference JIM 96, France, Vol. 4 (1996), 2-7.

[2] Ballot, C., On a family of recurrences that includes the Fibonacci and the Narayana recurrence, https://arxiv.org/abs/1704.04476v1.

[3] Biglici, G., The generalized order- $k$ Narayana cow's numbers, Math. Slovaca, Vol. 66(4) (2016), 795-802.

https://doi.org/10.1515/ms-2015-0181

[4] Didkivska, T.V., St'opochkina M.V., Properties of Fibonacci-Narayana numbers, In the World of Mathematics, Vol. 9(1) (2003), 29-36. (in Ukrainian)

[5] Flaut, C., Shpakivskyi, V., On generalized Fibonacci quaternions and FibonacciNarayana quaternions, Adv. Appl. Clifford Algebras, Vol. 23 (2013), 673-688. https://doi.org/10.1007/s00006-013-0388-2

[6] Koshy, T., Fibonacci and Lucas Numbers with Applications, John Wiley, New York, 2001.

https://doi.org/10.1002/9781118033067 
[7] Merca, M., A note on the determinant of a Toeplitz-Hessenberg matrix, Spec. Matrices, Vol. 1 (2013), 10-16.

https://doi.org/10.2478/spma-2013-0003

[8] Muir, T., The Theory of Determinants in the Historical Order of Development. Vol. 3, Dover Publications, New York, 1960. https://doi.org/10.2307/3604991

[9] Ramírez, J.L., Sirvent, V.F., A note on the $k$-Narayana sequence, Ann. Math. Inform., Vol. 45 (2015), 91-105.

[10] Sloane, N.J.A., The On-Line Encyclopedia of Integer Sequences, https://oeis. org.

[11] Zatorsky, R., Goy, T., Parapermanents of triangular matrices and some general theorems on number sequences, J. Integer Seq., Vol. 19 (2016), Article 16.2.2. 